\title{
Plasma properties of suprathermal electrons near comet 67P/Churyumov-Gerasimenko with Rosetta
}

\author{
M. Myllys ${ }^{1}$, P. Henri ${ }^{1}$, M. Galand ${ }^{2}$, K. L. Heritier ${ }^{2}$, N. Gilet ${ }^{1}$, R. Goldstein ${ }^{3}$, A. I. Eriksson ${ }^{4}$, \\ F. Johansson ${ }^{4}$, and J. Deca ${ }^{5,6}$
}

\author{
${ }^{1}$ Laboratoire de Physique et Chimie de l'Environnement et de l'Espace (LPC2E), CNRS, Orléans, France \\ e-mail: minna.myllys@cnrs-orleans.fr \\ 2 Department of Physics, Imperial College London, Prince Consort Road, London, SW7 2AZ, UK \\ ${ }^{3}$ Southwest Research Institute, PO Drawer 28510, San Antonio, TX 78228-0510, USA \\ ${ }^{4}$ Swedish Institute of Space Physics, Uppsala, Sweden \\ ${ }^{5}$ Laboratory for Atmospheric and Space Physics (LASP), University of Colorado Boulder, USA \\ ${ }^{6}$ Institute for Modeling Plasma, Atmospheres and Cosmic Dust, NASA/SSERVI, USA
}

Received 21 December 2018 / Accepted 21 May 2019

\begin{abstract}
Context. The Rosetta spacecraft escorted comet 67P/Churyumov-Gerasimenko from 2014 to September 2016. The mission provided in situ observations of the cometary plasma during different phases of the cometary activity, which enabled us to better understand its evolution as a function of heliocentric distance.

Aims. In this study, different electron populations, called warm and hot, observed by the Ion and Electron Sensor (IES) of the Rosetta Plasma Consortium (RPC) are investigated near the comet during the escorting phase of the Rosetta mission.

Methods. The estimates for the suprathermal electron densities and temperatures were extracted using IES electron data by fitting a double-kappa function to the measured velocity distributions. The fitting results were validated using observations from other RPC instruments. We give upgraded estimates for the warm and hot population densities compared to values previously shown in literature. Results. The fitted density and temperature estimates for both electron populations seen by IES are expressed as a function of heliocentric distance to study their evolution with the cometary activity. In addition, we studied the dependence between the electron properties and cometocentric distance.

Conclusions. We observed that when the neutral outgassing rate of the nucleus is high (i.e., near perihelion) the suprathermal electrons are well characterized by a double-kappa distribution. In addition, warm and hot populations show a significant dependence with the heliocentric distance. The populations become clearly denser near perihelion while their temperatures are observed to remain almost constant. Moreover, the warm electron population density is shown to be strongly dependent on the radial distance from the comet. Finally, based on our results we reject the hypothesis that hot electron population seen by IES consists of solely suprathermal (halo) solar wind electrons, while we suggest that the hot electron population mainly consists of solar wind thermal electrons that have undergone acceleration near the comet.
\end{abstract}

Key words. methods: data analysis - comets: general - plasmas

\section{Introduction}

When a comet travels through interplanetary space and approaches the Sun, its surface temperature increases and volatiles, such as water and carbon dioxide, start sublimating to create an expanding atmosphere around the comet. When such atmosphere gets ionized by solar extreme ultraviolet (EUV) radiation, electron-impact ionization and charge exchange with solar wind ions (Galand et al. 2016; Simon Wedlund et al. 2016), a cometary ionosphere forms around the comet. The cometary ionosphere has been observed to be a dynamic environment that undergoes changes as the position of the comet varies with respect to the Sun. Escorting comet 67P/Churyumov-Gerasimenko (67P) for more than two years, from 2014 until the end of September 2016, the European Space Agency (ESA)/Rosetta mission offered a unique opportunity to observe the evolution of the cometary plasma environment, as the plasma instruments on board ESA/Rosetta monitored the characteristics of the cometary plasma at different steps of the comet activity cycle.

The Rosetta Plasma Consortium (RPC) consisted of four plasma instruments together with a magnetometer and a Plasma Interface Unit (PIU) that were designed to provide in situ measurements of the cometary plasma environment around comet 67P (Carr et al. 2007). The Mutual Impedance Probe (RPC-MIP; Trotignon et al. 2007) and two Langmuir probes (RPC-LAP; Eriksson et al. 2007) monitored the cometary plasma environment, while the 3D energy distribution of the electrons was measured by the Ion and Electron Sensor (RPC-IES; Burch et al. 2007). The lowest energy threshold of RPC-IES was $4.32 \mathrm{eV}$ and even higher when the spacecraft potential was highly negative (Odelstad et al. 2015).

Throughout the escorting phase of Rosetta, high-energy electrons $(>10 \mathrm{eV})$ were observed near comet $67 \mathrm{P}$ by RPCIES. These high-energy electrons are also called suprathermal 
electrons (Clark et al. 2015; Madanian et al. 2016a; Broiles et al. 2016a) because their velocity distribution is not Maxwellian. Instead the electron population must be described by nonequilibrium distributions (Clark et al. 2015; Broiles et al. 2016a,b). However, the bulk electron population $(E \approx 5$ to $10 \mathrm{eV})$ may not have been thermalized at large heliocentric distances either. Since part of the bulk electron population have energies lower than the RPC-IES energy threshold, we are not able to confirm if the bulk population is described by a Maxwellian distribution.

Electrons with energies higher than the ionization potential can ionize the cometary neutrals and thus have a direct effect on the formation of the cometary ionosphere (Galand et al. 2016). The suprathermal electron fluxes at comet $67 \mathrm{P}$ are very dynamic and, in particular, they have been observed to increase rapidly as a response to the interaction with transient solar wind events, such as interplanetary corotating interaction regions (CIR; Edberg et al. 2016a; Hajra et al. 2018) and coronal mass ejections (CMEs; Edberg et al. 2016b). Hajra et al. (2018) studied four CIR events during the last four months (from June 2016 to September 2016) of the Rosetta mission. The authors showed that suprathermal electron fluxes as well as the total plasma density were greatly enhanced when CIRs reached the comet. Heritier et al. (2018) modeled the effect of photoionization and electronimpact ionization to the cometary plasma and they found that during two of the CIR intervals studied by Hajra et al. (2018) the electron-impact ionization frequency was increased together with the plasma density. On the other hand, the photoionization frequency was much lower than the electron-impact ionization frequency during the density peaks. Thus, Heritier et al. (2018) concluded that the main driver of the plasma enhancement during the CIR periods was electron-impact ionization, which had increased owing to high ionizing electron fluxes.

Even though the contribution of suprathermal electrons to the cometary plasma through electron-impact ionization has been studied and acknowledged, the source of these suprathermal electrons is still not clear. Madanian et al. (2016a) suggested that electrons are accelerated toward the comet by an ambipolar electric field. The ambipolar electric field results from the electron pressure gradient near the comet because of the strong plasma density inhomogeneity (Edberg et al. 2015). The electrons that are born near the comet are expected to be trapped inside the associated potential well. However, the external (i.e., solar wind) electrons or cometary electrons that are born far away from the comet are expected to be accelerated toward the nucleus as a consequence of the potential structure created by the ambipolar electric field. The existence of such a potential well creates an accelerated electron population near the comet that has higher energies than the cometary photoelectrons.

Deca et al. (2017) applied 3D fully kinetic and electromagnetic simulations of the solar wind interaction with a weakly outgassing comet. Because the dynamics of cometary ions and electrons were modeled self-consistently with the solar wind protons and electrons, the authors were able to study the origin of the electrons near the comet. The simulations by Deca et al. (2017) showed support for the ambipolar electric field scenario. According to the simulations the suprathermal electron population near the comet was identified to originate from the solar wind.

While the total electron density near comet $67 \mathrm{P}$ was measured by RPC-MIP through the plasma resonance at the plasma frequency, the suprathermal electron density needs to be extracted from the RPC-IES data. The suprathermal electron moments have been previously studied by Broiles et al. (2016a) using RPC-IES electron measurements. They estimated the plasma moments by fitting a double-kappa function on electron velocity distributions. Their fitting study covered two days: the first representing low cometary activity and the second for high activity. Broiles et al. (2016a) described the plasma observed by RPC-IES to be a sum of two electron populations, called warm and hot. The two populations are characterized by density, temperature, and an invariant kappa index. The warm population was observed to be dense, while the hot population was rarefied. Broiles et al. (2016a) speculated that the hot population could be the solar wind halo electrons (Pierrard et al. 2016), but no clear origin for the warm population was identified by the authors.

In a follow-up study, Broiles et al. (2016b) adopted the fitting method to a one-day interval in November 2014 and focused on the warm population. It was found that during the selected day, the warm population seemed to consist of two subpopulations: electrons with temperature above $8.6 \mathrm{eV}$ and below $8.6 \mathrm{eV}$. The population with higher energies were dominant in local neutral density compression regions while the cooler population dominated the local neutral rarefaction regions. Broiles et al. (2016b) discussed the possible heating and acceleration mechanisms of the two sub-populations. The possible mechanism of creating the subpopulation with a temperature above $8.6 \mathrm{eV}$ was proposed to be related to the wave-particle interactions and especially the heating due to lower hybrid waves.

In this paper, we study the warm and hot electron populations near the comet 67P introduced by Broiles et al. (2016a) over the full cometary phase of the Rosetta mission to characterize the evolution of these two populations. Sections 2 and 3 summarize the data sources and fitting routine. The fitted plasma parameters have been validated by comparing them with measurements from the other RPC instruments in appendix. Section 4 focuses on results of mapping the warm and hot electrons near the comet 67P. Density and temperature estimates for warm and hot populations are expressed as a function of radial distance from the Sun. The warm population is also observed to display a similar dependence of the cometocentric distance as the bulk electron population near comet. Discussion in Sect. 5 aims to explain the observed results in the context of current knowledge. Finally the findings are summarized in the conclusions.

\section{Data sources}

The study reported in this paper make use of data from the RPC-IES (Burch et al. 2007), RPC-MIP (Trotignon et al. 2007), and RPC-LAP (Eriksson et al. 2007) instruments. The RPCIES consisted of two electrostatic analyzers: one for measuring the $3 \mathrm{D}$ particle fluxes of ions and the other for electrons. The electron distribution measurements by RPC-IES set the basis of this study. The data treatment to convert the measured electron counts to physical quantities is explained in Sect. 3.2.

Data from the RPC-MIP instrument, in particular the plasma density, are used to compare and validate the suprathermal electron properties determined using the RPC-IES measurements. The RPC-MIP consisted of several transmitting and receiving electric antennas. The basic principle of the instrument is to measure the transfer electric impedance between the transmitters and receivers immersed in a plasma; an electric field is induced through the transmitters. The RPC-MIP operated in a frequency range from $7 \mathrm{kHz}$ to $3500 \mathrm{kHz}$. The mutual impedance indicated a resonance close to the plasma frequency that can be used to estimate the plasma density. The plasma density around the comet $67 \mathrm{P}$ is the main product of the RPC-MIP. There were two main operational modes in the RPC-MIP instrument called short Debye length (SDL) and long Debye length (LDL). The modes 
are used to measure plasma in different density conditions. The RPC-MIP was able to measure plasmas that have Debye length smaller than half of the distance between the receiving and transmitting electrodes (Trotignon et al. 2007), that is $40 \mathrm{~cm}$. Thus, in SDL mode the maximum measurable Debye length was $20 \mathrm{~cm}$. The LDL mode, on the other hand, used one of the LAP probes as a transmitter and thus the threshold for the measurable Debye length was increased to $2 \mathrm{~m}$ and hence, RPC-MIP could measure lower densities. The instrument could only operate one mode at the time and the derived plasma density is combination of measurements done in SDL and LDL modes.

Finally, data from the RPC-LAP instrument, in particular the spacecraft potential and the electron temperature (see Appendix A), are used, respectively, to derive the electron moments within the RPC-IES data treatment and to validate the plasma moments derived from RPC-IES measurements.

The RPC-LAP instrument consisted of two spherical Langmuir probes located at the end of two booms pointing outward of the spacecraft. The RPC-LAP measurements are based on detecting the current flowing to the probe. The current depends on the plasma density and the energy distribution of the surrounding plasma. See Odelstad et al. (2017) and Eriksson et al. (2017) for more information about the spacecraft potential and the electron temperature determination.

\section{Methods}

\subsection{Overview of RPC-IES electron data and derivation of phase-space density}

The electron part of the RPC-IES instrument was designed to observe the 3D energy distribution of the electrons (Burch et al. 2007). The energy range of the instrument went from $4.32 \mathrm{eV}$ to $17.67 \mathrm{keV}$ and all the 124 energy steps are used in this study. The elevation range covered 16 angle steps starting from $-48^{\circ}$ to $48^{\circ}$, which are the minimum and maximum elevation angles of the first and last bins. Thus, the center of the bins ranged from $-45^{\circ}$ to $45^{\circ}$. The azimuthal ranged from $0^{\circ}$ to $360^{\circ}$ using 16 anodes. Thus, the field of view (FOV) of the instrument was $96^{\circ} \times$ $360^{\circ}$. Because of telemetry constraints the measurements are not always returned using the highest possible grid but the adjacent azimuthal, elevation, and energy bins are summed together. The number of summed bins depends on the operational mode of the instrument. Thus, the energy and elevation resolution vary through out the study.

The accuracy of the measurements is influenced by spacecraft charging effects, which are monitored by the spacecraft floating electric potential. The negative spacecraft potential repels the lowest energy electrons to reach the instrument and increases the lowest effective energy threshold of the RPC-IES sensors (see Galand et al. 2016).

We started our analysis using the raw counts measured by RPC-IES (L2 production level publicly available on the ESA Planetary Science Archive (PSA ${ }^{1}$ ) to derive the phase-space density. The RPC-IES observed the amount of counts measured by each elevation, azimuthal, and energy bins. Heritier (2018) has provided a detailed description of deriving the differential energy flux (DEF) from the RPC-IES counts, which we have followed in this work.

To convert the RPC-IES counts $(C)$ to DEFs, the background counts $\left(C_{\mathrm{BG}}\right)$ caused by the penetrating radiation and electronic

\footnotetext{
1 https://www . cosmos.esa.int/web/psa/
}

thermal noise must be subtracted from the raw counts $(C)$ (Heritier 2018). The next step is to divide the remaining counts by the time interval that was used to gather the particle counts $(\Delta t)$ by the geometric factor $(G)$ and the by instrument efficiency $(\epsilon)$ (Broiles et al. 2016a; Heritier 2018) as expressed in the following formula:

$\mathrm{DEF}=\frac{C-C_{\mathrm{BG}}}{G \epsilon \Delta t}$

We used the in-flight geometric factor derived by Broiles et al. (2016a) that is energy and direction dependent.

\subsection{Fitting method}

We start by summarizing our fitting approach.

The fitting procedure starts by converting the measured electron DEF to velocity phase-space density. Before the conversion from DEF to phase space, the DEF has been integrated over the observed directions and divided by integral over the observed angle (Heritier et al. 2018; Heritier 2018). When treating RPCIES data, it should be noted that spacecraft structure and other instruments blocked part of the theoretical RPC-IES FOV (Clark et al. 2015). In addition, the anode 11 did not give any data for instrumental reasons and anodes $8-15$ have been reported to measure reduced DEF at lower energies after April 2015 (Broiles et al. 2016a) possible due to a threshold change in one of its octal amplifiers. Thus, we consistently used measurements only from the anodes 0-7 with Broiles et al. (2016a,b). We also assume the plasma is isotropic and multiply the space averaged DEF by $4 \pi$ to get the phase-space density as shown below (Heritier 2018):

$f(v)_{\text {observed }}=\frac{2}{v^{4}} \times 4 \pi \frac{\int_{180^{\circ}}^{360^{\circ}} \int_{-48^{\circ}}^{48^{\circ}} \operatorname{DEF}(v, \theta, \phi) \cos \theta \mathrm{d} \theta \mathrm{d} \phi}{\int_{180^{\circ}}^{360^{\circ}} \int_{-48^{\circ}}^{48^{\circ}} \cos \theta \mathrm{d} \theta \mathrm{d} \phi}$.

The resulting phase-space densities differ from those shown in Fig. 6 by Broiles et al. (2016a) and in Fig. 7 in Broiles et al. (2016b). Indeed, according to the articles, Broiles et al. (2016b) is only summing the space-phase density over all elevations and azimuths from $180^{\circ}$ to $360^{\circ}$, while in this work, the space averaged DEF is computed and multiplied by $4 \pi$. This explains why previously reported results were slightly lower than the phase-space density computed in this work.

We adopt the double-kappa function introduced by Broiles et al. (2016a) and fit this function to electron velocity distribution measured by RPC-IES. We fit the model function, double-kappa, to $f(v)_{\text {observed }}$ to get the estimate for the warm and hot population densities $\left(n_{\mathrm{W}}\right.$ and $\left.n_{\mathrm{H}}\right)$, temperatures $\left(T_{\mathrm{W}}\right.$ and $\left.T_{\mathrm{H}}\right)$, and invariant kappa indices $\left(\kappa_{\mathrm{W}}\right.$ and $\left.\kappa_{\mathrm{H}}\right)$. The time steps that fit poorly to the model function have been automatically removed by the fitting routine based on the reduced $\chi^{2}$ value $\left(0.5<\chi^{2}<170\right.$ are accepted) and other criteria that have been selected based on visual inspection of the fitting results (details are given in Sect. 3.3). The fitting technique searches estimates for the plasma parameters by minimizing the $\chi^{2}$ for every measured RPC-IES spectra using the Nelder-Mead method.

The fitted distribution function $(f)$ consists of three parts $f_{\mathrm{w}}+f_{\mathrm{h}}+f_{\mathrm{BG}}$, where $w$ refers to the warm population, $h$ to the hot population, and $B G$ to the background. The kappa distribution is using the invariant kappa index (Livadiotis \& McComas 2011). The distribution functions to all populations are written below (Broiles et al. 2016a). First, the distribution function of the warm 
populations is written as

$f(v)_{\mathrm{w}}=\frac{n_{\mathrm{w}}}{\left(\kappa_{\mathrm{w}} \pi\left(\frac{2 k_{\mathrm{B}} T_{w}}{m_{\mathrm{e}}}\right)\right)^{\frac{3}{2}}} \frac{\Gamma\left(\kappa_{\mathrm{w}}+5 / 2\right)}{\Gamma\left(\kappa_{\mathrm{w}}+1\right)}\left[1+\frac{(v-\langle v\rangle)^{2}+\frac{2 q \Phi_{\mathrm{SC}}}{m_{\mathrm{e}}}}{\kappa_{\mathrm{w}}\left(\frac{2 k_{\mathrm{B}} T_{\mathrm{w}}}{m_{\mathrm{e}}}\right)}\right]^{-\kappa_{\mathrm{w}}-\frac{5}{2}}$,

where $k_{\mathrm{B}}$ is the Boltzmann constant, $m_{\mathrm{e}}$ the electron mass, and $q$ the elementary charge.

Second, the hot population distribution is expressed with a form similar to the warm population, but in this case the spacecraft potential effect $\left(\Phi_{\mathrm{SC}}\right)$ and the bulk velocity $(\langle v\rangle)$ have both been neglected, as done by Broiles et al. (2016a). The spacecraft potential has a negligible effect on the hot population, i.e.,

$f(v)_{\mathrm{h}}=\frac{n_{\mathrm{h}}}{\left(\kappa_{\mathrm{h}} \pi\left(\frac{2 k_{\mathrm{B}} T_{\mathrm{h}}}{m_{\mathrm{e}}}\right)\right)^{\frac{3}{2}}} \frac{\Gamma\left(\kappa_{\mathrm{h}}+5 / 2\right)}{\Gamma\left(\kappa_{\mathrm{h}}+1\right)}\left[1+\frac{v^{2}}{\kappa_{\mathrm{h}}\left(\frac{2 k_{\mathrm{B}} T_{\mathrm{h}}}{m_{\mathrm{e}}}\right)}\right]^{-\kappa_{\mathrm{h}}-\frac{5}{2}}$.

Finally, the background distribution is also defined identically with the Broiles et al. (2016a),

$f_{\mathrm{BG}}=\frac{2 \times 1.2}{v^{4} G}$,

where $G$ the geometric factor is given in Burch et al. (2007).

The spacecraft potential has been taken into account in the warm population distribution function. This means that the potential term affects to the fitted $n_{\mathrm{w}}$ and $T_{\mathrm{w}}$ values, and especially to $n_{\mathrm{w}}$. If the spacecraft potential term were zero, the fitting routine would offer lower values for $n_{\mathrm{w}}$ for times when the potential was negative. Thus, the spacecraft potential in the distribution function compensates for the missing electrons that are not able to reach the instrument.

In Appendix $C$, we show that we get similar results when the spacecraft potential term is included to the fitted distribution function or the spacecraft potential correction is included in the RPC-IES space phase density directly, using the method by Galand et al. (2016), before the fitting is done, while setting the potential term in Eq. (3) to zero.

The spacecraft potential is estimated using the data from the RPC-LAP instrument (Odelstad et al. 2015). Time periods when spacecraft potential is lower than $-16 \mathrm{~V}$ are removed from the study. This is because we want to avoid times when the measured DEF is altered too much by the spacecraft potential. Furthermore, we removed the RPC-IES spectra from the study if the spacecraft potential estimate was not available. This approach differs from that of Broiles et al. (2016a), where the spacecraft potential was set to zero when it was not available. Indeed, setting the spacecraft potential to zero increases the risk of underestimating the density.

A comprehensive validation of the fitting results is shown in Appendix B. We validated our results by comparing the extracted warm electron density and temperature with independent measurements done contemporarily by RPC-MIP and RPC-LAP. In addition, the fitted RPC-IES warm electron density has been compared with integrated RPC-IES warm electron density to show that both methods give values that are in excellent agreement.

\subsection{Problems of the double-kappa fitting}

We ran the fitting procedure through the whole time interval when Rosetta was escorting the comet 67P, from summer 2014 until the end of the mission in September 2016. However, it was not possible to fit the double kappa for every measured RPC-IES spectra. There are several reasons for this.

To ensure the results are robust, we implemented the fitting routine to reject automatically part of the fitted parameters based on the following criteria: (1) the fitting method does not converge to a local minimum; (2) the reduced chi square is either much smaller $\left(\chi^{2}<0.5\right)$ than one and the routine is "over-fitting" the data or much higher $\left(\chi^{2}>170\right)$ than one, and the observed phase-space density poorly fits the double kappa; (3) the number of fitted data points is too low $(<20)$; $(4)$ the spacecraft potential by RPC-LAP is lower than $-16 \mathrm{~V}$ or is not available; (5) the warm population electron density is lower than the hot population density; and finally, (6) the warm population temperature is higher than the hot population. The spacecraft potential criterion removes almost $70 \%$ from the data points for $2014,23 \%$ in 2015 , and $48 \%$ in 2016 . Thus, the potential is the most limiting criterion.

The spacecraft potential criterion is also expected to exclude most of the time periods when the comet was interacting with large-scale solar wind structures such as CIRs when the spacecraft potential can become very low rapidly (Hajra et al. 2018). Since high-energy electron fluxes have been observed to increase greatly during CIR periods (Edberg et al. 2016a; Hajra et al. 2018), it is unclear whether the electron velocity distribution function still consists of two electron populations as is expected by the double kappa.

Figure 1 shows different examples of measured phase-space distributions $\left(f(v)_{\text {observed }}\right)$, together with the best-fitted curves, expressed in energy space. The data points are shifted in energy according to the measured spacecraft potential value. The blue curve indicates the warm population while the red curve is for the hot population. The black curve is the sum of the two populations and the constant background term. The upper panels (A, B, and $C$ ) are examples of times when the best-fitting results were accepted, while the lower panels (D, E, and F) are examples of best fits that are actually rejected in the rest of the study. All the distributions are taken from the same day (2015-12-17). In Fig. 1, the reduced $\chi^{2}$ decreases from left to right in the upper panels. The values are respectively 68.1, 21.6, and 8.4. Panels D and E show examples when the fitting has been rejected because of the $\chi^{2}$ values are too high: in both cases the $\chi^{2}$ is above 220 . Panel $\mathrm{F}$ shows a distribution when there is a small bump near the energy $10^{2} \mathrm{eV}$. These bumps appear in the distributions every now and then and they have negative impact to the fitting results. Panels E and $\mathrm{D}$ both show cases in which the function for the hot population tries to fit the low-energy part of the measured distribution where the warm population should actually be. Because we have no reason to believe that this would be physical, we automatically reject such results (criterion 6).

Criteria 1 and 2 mostly arise during times when the shape of the electron velocity distribution is not well described by double kappa. Figure 2 is showing how the times when the double-kappa distribution does not fit to the data are distributed throughout the studied time interval. The bars show the ratio of RPC-IES spectra that were rejected to all measured spectra during one month period. Time intervals when the fitting was not possible as a consequence of low spacecraft potential are excluded from the statistics. The red line in Fig. 2 shows the distances of Rosetta from the Sun (i.e., heliocentric distance). The cometary activity depends on the heliocentric distance because the outgassing of neutrals becomes stronger when the comet approaches the Sun and the cometary surface temperature rises. However, it should 

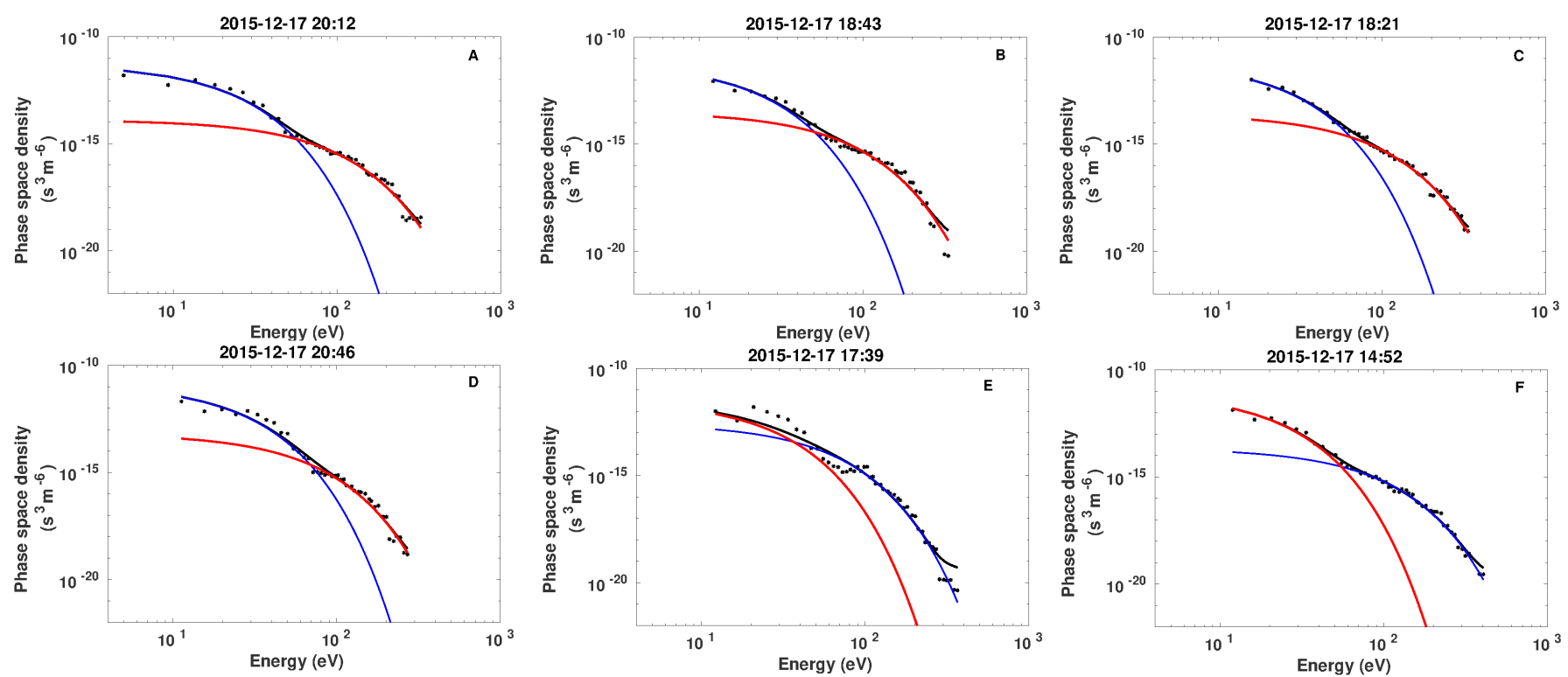

Fig. 1. Examples of the fitted distributions. The observed phase-space density as a function of energy is plotted with black dots, the fitted distribution for the warm population is plotted with blue, and the hot population with red. The black curves are the sum of the warm and hot population distributions and the constant background term. Upper panels: examples of distributions when the fitted parameters were accepted. Lower panels: indicate when the fitted parameters were rejected from the study. The $\chi^{2}$ values for the upper panels from left to right are 68.4, 21.6, and 8.4.

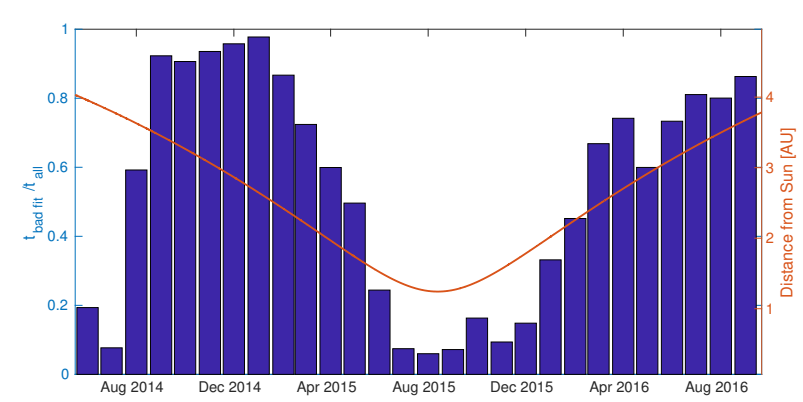

Fig. 2. Histograms showing the ratio of bad fits to all measured RPCIES spectra during one month period. The ratio is shown in left-hand side of the panel. The red line is the heliocentric distance of the spacecraft and its scale is given on the right-hand side.

be noted that the outgassing rate reached its maximum around a month after perihelion (Hansen et al. 2016).

As can be seen from the Fig. 2, there is clear asymmetry in the fitting results. During 2015 near perihelion only around $10 \%$ of the time double-kappa function is rejected by the fitting routine. This means that $90 \%$ of the time a double-kappa function represents a good description of the shape of the electron velocity distribution. However, at a large heliocentric distance the situation can be the opposite and the double-kappa function might not be considered the best representation of the electron distribution the majority of the time.

There are several possible reasons why the fitting routine gives poorer results for 2014 and 2016 compared to 2015. For example, in 2014 the data is characterized by a higher level of background than in 2016, even after the background counts are subtracted. This might affect the fitting results especially regarding the properties of the hot electron population. The hot population is more difficult to fit at large heliocentric distances because the population is less dense and it might be shielded by the suprathermal tail of the warm population itself. It is also possible that the electron distributions are less stable or would fit better to some other distribution function during low cometary activity phases. We also assume isotropy in the fitting process and that can affect the fitting results. If the electron distributions are more asymmetric at large distances from the Sun, for example, because of some heating or acceleration process, which would lead to poorer fitting results as well.

\section{Results}

Based on the robust data treatment described above, computed during the whole cometary phase of the Rosetta mission, we now concentrate on the properties of the warm and hot electron populations observed at comet $67 \mathrm{P}$.

\subsection{Suprathermal electron properties as a function of heliocentric distance}

The double-kappa fitting procedure was run over the whole time interval when Rosetta was escorting the comet and the fitted parameters were divided into bins based on the heliocentric distance where the measurements were taken. The width of the bin is $0.3 \mathrm{AU}$ and the binning range goes from 1.2 to $3.9 \mathrm{AU}$. The neighboring bins are partly overlapping.

The median of a density for hot (red dots) and warm (blue dots) populations inside each bin are shown in Fig. 3. The error bars are showing the first and the third quartiles to describe the spread of the data inside each bin. The warm population median density observed at the location of the Rosetta orbiter spacecraft is around $100 \mathrm{~cm}^{-3}$ and it shows only a weak dependence with the heliocentric distance (which essentially reflects the fact that the Rosetta orbiter was moved away from the comet nucleus during high cometary outgassing activity, for safety reasons.) while the hot population median density is clearly decreasing with heliocentric distance. Near perihelion the hot population is observed to be around $3 \mathrm{~cm}^{-3}$ while at $3.5 \mathrm{AU}$ the density is only $0.1 \mathrm{~cm}^{-3}$.

Figure 4 is showing the warm and hot population median temperatures as a function of heliocentric distance. The 


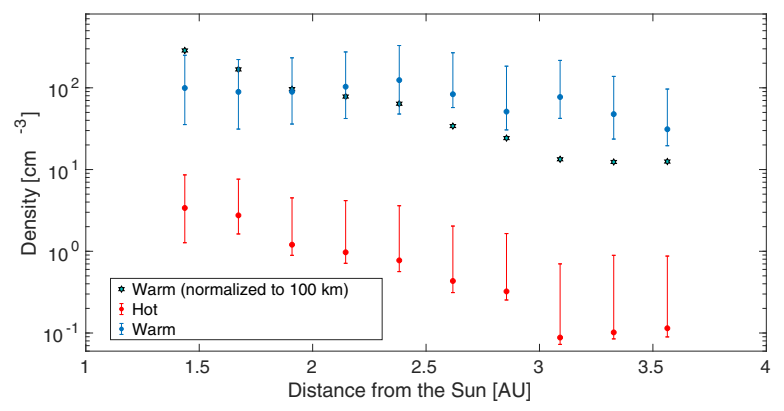

Fig. 3. Hot (red dots) and warm (blue dots) electron population densities as a function of heliospheric distance. The blue stars shows the warm population density that has been normalized to $100 \mathrm{~km}$ (see Sect. 5.2). The error bars are showing the first and the third quartile to describe the spread of the data inside each bin

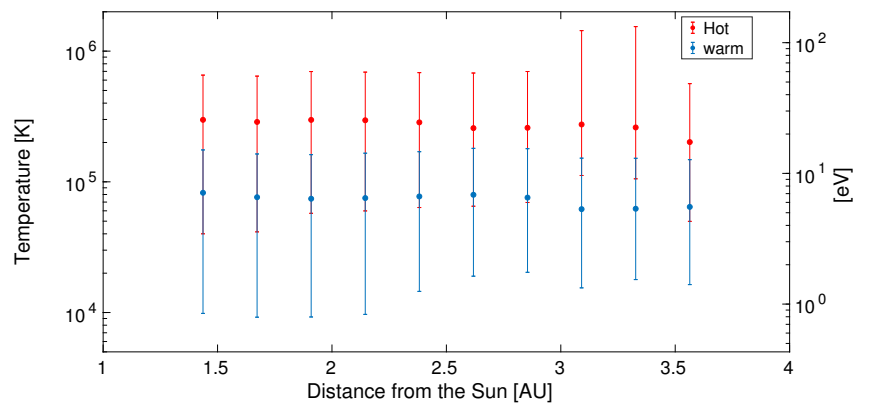

Fig. 4. Hot and warm electron population temperatures as a function of heliospheric distance. The error bars are showing the first and the third quartile to describe the spread of the data inside each bin.

temperature for the warm and hot populations stays almost constant whatever the heliocentric distance. The typical temperature for the hot (warm) population is around $20 \mathrm{eV}(6 \mathrm{eV})$.

\subsection{Suprathermal electron properties as a function of cometocentric distance}

Edberg et al. (2015) studied how the electron density near the comet $67 \mathrm{P}$ varies as a function of cometocentric distance $\left(r_{\mathrm{c}}\right)$. The authors used the electron density measurements by RPC-LAP from 4 to 28 February 2015 when Rosetta did two cometary flybys to measure the vertical profile of plasma density. When the spacecraft moved farther away from the comet the density is consistent with a $1 / r_{\mathrm{c}}$ drop, as is expected for the bulk of the electrons from the theory at large heliocentric distance (Galand et al. 2016; Beth et al. 2019) and confirmed from multi-instrument studies (Heritier et al. 2017, 2018). We used the same period of time as Edberg et al. (2015) to study the cometocentric dependence of the warm and hot electron populations. Such flybys are indeed ideal to study the vertical profile of electron density because cometary outgassing activity does not vary much during such a short time interval.

Densities for both populations as a function of cometocentric distance are shown in Fig. 5; see also Fig. B.5, which shows the density comparison between RPC-MIP and fitted RPC-IES. The upper panel (A) indicates the warm population and lower panel (B) indicates the hot population. The blue points show the fitted density values for individual time steps, while the red dots represent the median values for $30 \mathrm{~km}$ altitude intervals. The intervals are partly overlapping as illustrated in the left corner in Fig. 5A. The black curve shows a result when a power
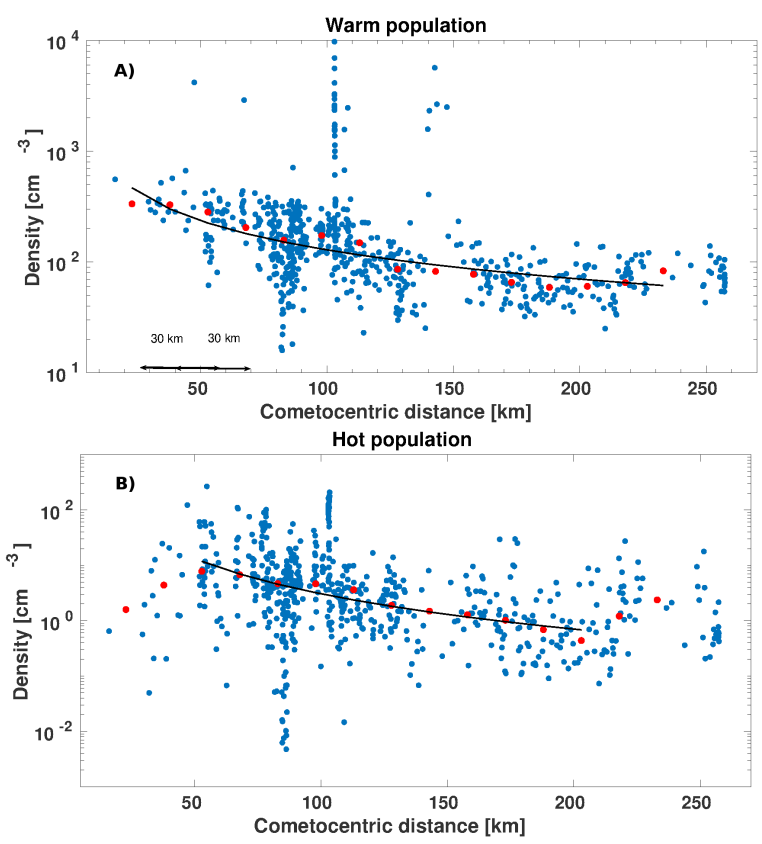

Fig. 5. Warm (panel $A$ ) and hot (panel B) population densities as a function of cometocentric distance between 4 and 28 February 2015. The blue points show all the fitted densities while the red dots show the $30 \mathrm{~km}$ medians of the data. The black line indicate the power law fit to the median values. Only $11 \%$ of the measured RPC-IES spectra were successfully fitted during the studied time interval.

law function was fitted to the median values. The fitting gives $1 / r_{\mathrm{c}}^{1.2}$ dependence for the curve. This differs slightly for the $1 / r_{\mathrm{c}}$ dependence for the total electron density but it may indicate that the warm population consists partly of bulk electrons (i.e., electrons created during the ionization processes). However, worthy of remark is that there is scatter in the density data and $r_{\mathrm{c}}^{-1.2}$ is a rough estimate of how the density is decreasing as a function of cometocentric distance. The data is indeed composed of measurements made at different latitudes and longitudes that have different local outgassing rates, which directly affects the observed scatter.

Figure 5B shows the cometocentric dependence for the hot electron population. The median values have been computed using the same grid as for the warm population density. The two median values at low altitudes $(<50 \mathrm{~km})$ are clearly decreased compared to the values between 50 and $100 \mathrm{~km}$. This is because the number of data points (i.e., fitted density estimates) is really low when Rosetta is near the comet. In this region the warm population becomes denser and based on visual inspection it can shield the hot population in the measured phase-space density distribution. Thus, the fitting gives very low values (near zero or even negative) for the hot population density and temperature, and it is not possible to get physically meaningful estimates for the parameters. Also, when the altitude is higher than $210 \mathrm{~km}$ the last two medians are greatly affected by the lack of data points in the region. Thus, the power law fitting has been made excluding the median values with the lowest and highest altitudes and it gives $1 / r_{\mathrm{c}}^{2.1}$ dependence for the density. This clearly differs from the $1 / r_{\mathrm{c}}$ dependence that is observed for the total electron content.

We also used the RPC-IES measurements during the dayside excursion (from 2015-08-01 to 2015-10-20) to study the cometocentric distance dependence to see if we are able to reproduce the same behavior as during the flybys in February 


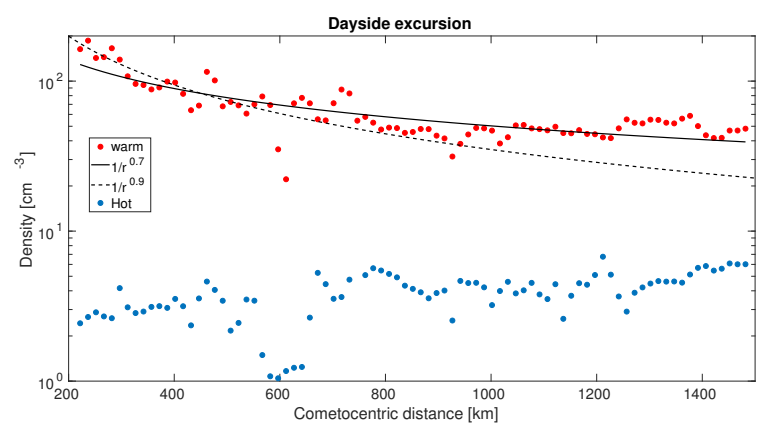

Fig. 6. Warm and hot population densities as a function of cometocentric distance between 2015-08-01 and 2015-10-20. The red dots show the $30 \mathrm{~km}$ medians of the data. The black solid line shows the power law fit to the median values using the whole cometocentric distance range, while the black dashed line indicates a power law fit to points when $r_{\mathrm{c}}<$ $450 \mathrm{~km} .86 \%$ of the measured RPC-IES spectra were successfully fitted during the studied time interval.

2015. A CME occurred during the dayside excursion (Edberg et al. 2016b), but the fitted densities during the CME interval have been removed. The medians for the warm (red) and hot (blue) population densities are shown in Fig. 6.

The power law fitting was done in the case of the warm population. The black solid line shows the power law fitting using the whole cometocentric distance range, while the black dotted line was created by using only altitudes below $450 \mathrm{~km}$. The black solid line follows a dependence in $1 / r^{0.7}$ and estimates the warm population density value at large heliocentric distances better than the dotted line. However, the black solid line underestimates the density value near the comet. The dotted line follows a trend in $1 / r^{0.9}$ that is close to the $1 / r^{1}$ dependence for the bulk electron populations, but underestimates the density value at large distances $(>900 \mathrm{~km})$.

It appears that densities for both the warm and hot populations saturate at high cometocentric distances during the dayside excursion. The warm population saturates after $800 \mathrm{~km}$, while the hot population stays nearly constant during the studied altitude range, excluding values near $600 \mathrm{~km}$ that are decreased for an unknown reason. It seems that the warm population density follows the $1 / r_{\mathrm{c}}$ trend near the comet but the behavior changes at large cometocentric distances $(>900 \mathrm{~km})$.

Figure 5 covers the cometocentric distance range only up to $250 \mathrm{~km}$ while Fig. 6 begins after $200 \mathrm{~km}$. The outgassing rate during the time intervals presented in Figs. 5 and 6 varies and thus the amplitudes of the densities are not directly comparable. Nonetheless, both figures confirm that when the distance from the comet is less than $450 \mathrm{~km}, 1 / r_{\mathrm{c}}^{1}$ is a valid approximation of the cometocentric distance dependence for the warm population.

However, the hot population seems to have different behavior during the February 2015 flybys (Fig. 5) and the dayside excursion (Fig. 6). During February 2015, the hot population seems to drop as $1 / r_{\mathrm{c}}^{2}$. Even though the power law dependence in $1 / r_{\mathrm{c}}^{2}$ for the hot population density might seem to occur because some unknown process is generating the hot population near the comet surface which is then expanding spherically to the surroundings, this scenario is unlikely. On the other hand, the hot population would be born near the comet, its density should increase rapidly when $r_{\mathrm{c}}$ is less than $50 \mathrm{~km}$, and the ratio of the hot to warm population densities should increase. This should make it easy to fit the hot population near the comet which differs from the case mentioned earlier. On the other hand, in Fig. 6 during the dayside
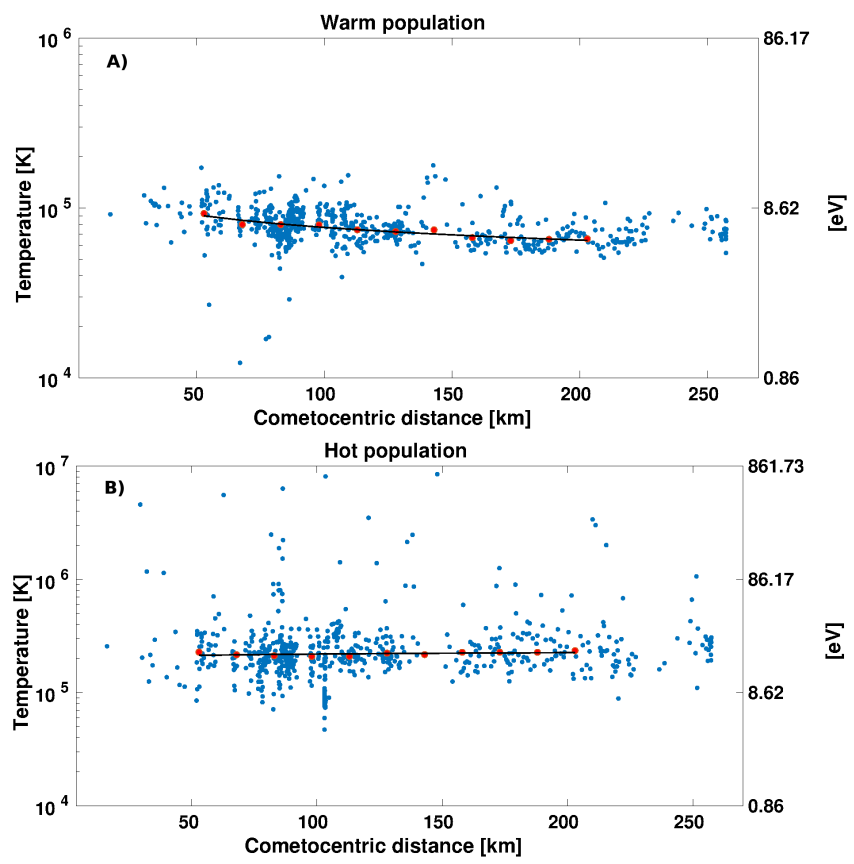

Fig. 7. Warm (panel $A$ ) and hot (panel B) population temperatures as a function of cometocentric distance between 4 and 28 February 2015. The blue points show all fitted temperatures while the red dots show the $30 \mathrm{~km}$ medians of the data. The black line indicates the power law fit to the median values.

excursion the hot population is not following the $1 / r_{\mathrm{c}}^{2}$ trend anymore, but the density stays quite constant or even increased a little when the spacecraft altitude became higher. Thus, it is not possible to draw a clear conclusion how the hot population depends on the cometocentric distance.

While warm and hot population densities show a dependence with the cometocentric distance, their temperatures show no clear trend (see Fig. 7). Both populations have nearly constant temperatures, namely $7 \times 10^{4} \mathrm{~K}(\approx 6 \mathrm{eV})$ for the warm and $2 \times 10^{5} \mathrm{~K}(\approx 17 \mathrm{eV})$ for the hot populations.

The warm population density at a given cometocentric distance $r_{\mathrm{c}}$ can be expressed as $n_{\mathrm{w}}\left(r_{\mathrm{c}}\right)=\left(R / r_{\mathrm{c}}\right) n_{\mathrm{wf}}$, where $n_{\mathrm{wf}}$ is the density given by the fitting procedure at the cometocentric distance $R$ of Rosetta. The density in Fig. 3 is replotted, taking into account the observed dependence with cometocentric distance where the RPC-IES spectra was measured and normalizing the density to an altitude $\left(r_{\mathrm{c}}\right)$ of $100 \mathrm{~km}$. The results are shown in Fig. 3 along with the non-normalized densities. The blue stars show the cometocentric distance corrected warm population densities. After taking into account the cometocentric dependence, the warm population density shows a clear decreasing trend with the distance to the Sun. The decreasing trend can be understood to be caused by the change in the cometary activity and the outgassing rate (Hansen et al. 2016) as well as varying ionization frequency (Heritier et al. 2018).

\subsection{Comparison between the solar wind and RPC-IES electron populations}

The undisturbed solar wind electron distribution can usually be decomposed by a solar wind core electron population that is well described by a Maxwellian distribution and by a suprathermal electron tail that has two components: halo and strahl (Feldman et al. 1975; Fitzenreiter et al. 1998; Štverak et al. 2009). The halo electrons are expected to be generally isotropic 
while the strahl component is a magnetic field-aligned beam. Pierrard et al. (2016) studied core and halo properties by fitting a combination of Maxwellian and Kappa distributions to solar wind electron observations collected by three different spacecraft at various heliocentric distances. The RPC-IES electron densities and temperatures shown in Figs. 3 and 4 can be compared with those of undisturbed solar wind thermal core and suprathermal halo electron properties available from the literature (Pierrard et al. 2016).

At 1.4 AU the thermal core electrons have average densities around $3 \mathrm{~cm}^{-3}$ (Pierrard et al. 2016) while the warm population density is around $300 \mathrm{~cm}^{-3}$ (Fig. 3) when the density is normalized to an altitude of $100 \mathrm{~km}$. Hence, the warm electron density is about 100 times higher than the thermal core in the solar wind. At $3 \mathrm{AU}$, the difference between the solar wind core electrons and warm population density is lower but the warm population is still around around 22 times higher (core electrons: $0.69 \mathrm{~cm}^{-3}$, warm population: $15 \mathrm{~cm}^{-3}$ ). Hence, the majority of the RPCIES warm population electrons near the nucleus are likely to be of cometary origin, composed of a mixed of bulk and accelerated electrons. This agrees with the bulk of the plasma close to the nucleus to be of cometary origin (e.g., Galand et al. 2016; Heritier et al. 2018).

If we compare the hot population density observed in the vicinity of comet $67 \mathrm{P}$ at $1.4 \mathrm{AU}$ with the undisturbed solar wind core electrons at $1.4 \mathrm{AU}$, interestingly, the densities are more or less the same (see Fig. 8). At $3 \mathrm{AU}$, the undisturbed solar wind core is around three times higher than the hot population density observed in the vicinity of comet $67 \mathrm{P}$, but the core electron density is still well within the uncertainty range of the hot population density. It seems that the hot population follows the trend of undisturbed solar wind core electron density. On the other hand, the density for the suprathermal halo electrons in the undisturbed solar wind varies from 0.26 to $0.02 \mathrm{~cm}^{-3}$ between 1.4 and $3 \mathrm{AU}$ meaning that it is always much less dense than the hot population.

We now compare the temperatures between the two electron populations near the comet observed by RPC-IES with the undisturbed solar wind electron populations. The solar wind core electron temperature decreases from $1.1 \times 10^{5} \mathrm{~K}(\approx 9 \mathrm{eV})$ to $0.86 \times 10^{5} \mathrm{~K}(\approx 7 \mathrm{eV})$ and halo electron temperature from $2 \times 10^{5} \mathrm{~K}(\approx 17 \mathrm{eV})$ to $1 \times 10^{5} \mathrm{~K}(\approx 9 \mathrm{eV})$, while the heliocentric distance increases from 1.4 to 3 AU (Pierrard et al. 2016). As can be seen from Fig. 4 the warm population is always colder than the solar wind core and halo electrons, while the hot population is hotter than the core and clearly colder than the halo electrons. The difference between the solar wind thermal core electron temperature and hot population temperature at $1.4 \mathrm{AU}$ is around $1.9 \times 10^{5} \mathrm{~K}(\approx 16 \mathrm{eV})$ and $1.64 \times 10^{5} \mathrm{~K}(\approx 14 \mathrm{eV})$ at $3 \mathrm{AU}$.

\section{Discussion}

\subsection{Suprathermal electron observations near comet $67 P$}

The suprathermal electrons near the comet 67P observed by Rosetta have been previously studied by several authors. Madanian et al. (2016a) studied the suprathermal electron environment using measurements from year 2014 when the distance from the Sun was around $3 \mathrm{AU}$. The authors pointed out that the observed electron fluxes near the comet exceeded greatly typical solar wind values. Madanian et al. (2016a) assumed isotropic temperatures and integrated the electron densities over the whole RPC-IES energy range for a 13-day interval. The integrated electron densities varied between 10 and $100 \mathrm{~cm}^{-3}$ but the highest

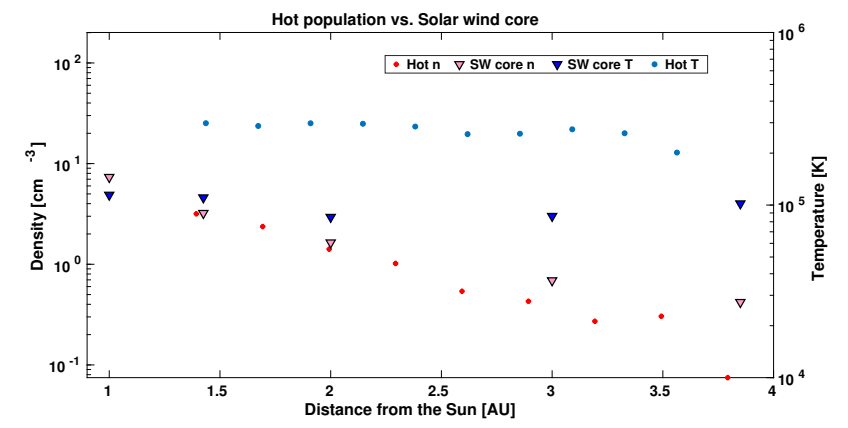

Fig. 8. Hot population density (red dots) and temperature (blue dots) as a function of heliocentric distance. The solar wind core population density is shown with yellow triangles and temperatrue with black triangles. The values for solar wind core populations are taken from Pierrard et al. (2016).

values were achieved during a CME interaction. The RPC-IES electron densities achieved by Madanian et al. (2016a) are about one order of magnitude lower than the total electron density measured by RPC-LAP and RPC-MIP. The average warm population density in our study at $3 \mathrm{AU}$ is around $80 \mathrm{~cm}^{-3}$, which is also higher than the values shown by Madanian et al. (2016a).

There are some explanations for the difference between the densities reported in this study and given by Madanian et al. (2016a). First, we used the in-flight geometrical factor, which was released after Madanian et al. (2016a) was published, to compute the electron fluxes from the counts measured by RPC-IES. In turn Madanian et al. (2016a) have used the geometrical factor given by Burch et al. (2007). We also included the instrument efficiency $(\epsilon)$ to the flux calculation, which has not been taken into account by Madanian et al. (2016a; see their Eq. (2)). Also, the spacecraft potential effect to the electron fluxes are ignored in the integration by Madanian et al. (2016a). This can be a problem especially during the CME interaction when the spacecraft potential can be very negative and the bulk electron population is not measurable by RPC-IES. Hence, such integration can provide incorrect density values.

We adopted our fitted double-kappa distribution from the papers by Broiles et al. (2016a,b) and the fitting method have been summarized in Sect. 3.1. Broiles et al. (2016a,b) showed warm and hot population densities that are almost ten times lower than ours. For example, for a one-day interval when the comet was at heliocentric distance $3 \mathrm{AU}$ the warm population density by Broiles et al. (2016a) varied between 10 and $30 \mathrm{~cm}^{-3}$ and between 10 and $100 \mathrm{~cm}^{-3}$ at heliocentric distance $1.3 \mathrm{AU}$, while our warm population densities are systematically around $100 \mathrm{~cm}^{-3}$ or more. At 1.3 AU Broiles et al. (2016a) found that during one-day interval the warm population temperature varied between 5 and $10 \times 10^{4} \mathrm{~K}(\approx 4-9 \mathrm{eV})$ and the hot population temperature as $2-10 \times 10^{5} \mathrm{~K}(\approx 17-86 \mathrm{eV})$. Our temperature values (see Fig. 4) are in same ranges. However, there are differences in the temperature estimates at $3 \mathrm{AU}$. Broiles et al. (2016a) obtained higher values for the two populations than we have shown in this study. It is not clear from where such differences between our results and the previous results by Broiles et al. (2016a) arise. We are very confident with the densities and temperatures obtained with this updated data treatment given that we cross-validated our results with independent plasma density and temperature measurements made from two other Rosetta experiments; i.e., RPC-MIP and RPC-LAP, which are thoroughly described in the Appendix B. 


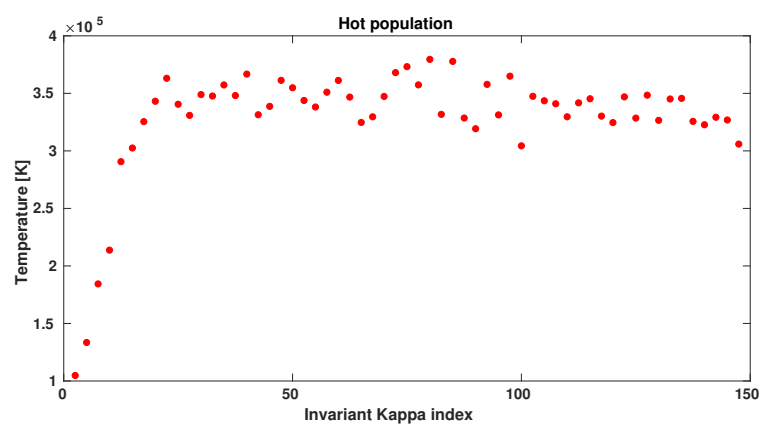

Fig. 9. Hot population temperature as a function of invariant kappa index.

\subsection{Origin of the suprathermal electrons}

The origin of the suprathermal electrons observed in the vicinity of comet $67 \mathrm{P}$ has been a key question during and after the Rosetta mission. Broiles et al. (2016a) suggested that hot population could be solar wind halo electrons. However, according to our analysis this hypothesis is unlikely to be true. We showed in Sect. 4.3 that the hot population is much denser and colder than solar wind halo component. The hot population density observed by RPC-IES is almost one order of magnitude higher than the suprathermal halo density in the solar wind.

Pierrard et al. (2016) studied how the parallel and perpendicular temperatures for the solar wind halo component vary as a function of the kappa index. Both temperature components showed decreasing trend with increasing kappa index. Figure 9 illustrates the temperature for the hot population as a function of the invariant kappa index. The data have been smoothed by dividing the invariant kappa index into bins and computing the median temperature inside each bin. As shown in Fig. 9, the hot population temperature increases with increasing kappa index, which contradicts the halo population temperature. Hence, we draw the conclusion that the hot population is not solely the solar wind halo component.

The solar wind core electron density is the same order of magnitude as the hot population density and both densities decrease with the increasing distance from the Sun, as is shown in Sect. 4.3. Thus, it might be possible that the hot population actually originates from the solar wind and accelerates near the cometary coma, for instance through the effect of the ambipolar electric field. Indeed, Madanian et al. (2016a) studied the effect of the ambipolar electric field, which is associated with the near-cometary density gradient, on the acceleration of electrons and suggested a scenario for the origin of the suprathermal electrons. According to this scenario, a potential well is created around the comet due to the density gradient, which can trap the electrons that are born near the comet. In turn, the potential well can accelerate the solar wind electrons toward the comet along the ambipolar electric field. This scenario was later tested by Deca et al. (2017) using 3D fully kinetic simulations of the cometary environment for low outgassing rates and noted that the suprathermal electrons near the comet actually originated from the solar wind core electron population itself.

The ambipolar electric field is able to accelerate electrons to energies that are few times the average kinetic energy $\left(\left\langle E_{\text {kin }}\right\rangle \approx 10 \mathrm{eV}\right)$ of the cometary bulk electrons (Madanian et al. 2016a). Since the peak value of the probability density function of the hot population energies is near $40-60 \mathrm{eV}$, depending on the time step, the ambipolar electric field is able to explain the energy increase of the solar wind core electrons. Owing to the acceleration, the solar wind electrons are also heated (Deca et al. 2017). Hence, if the hot population mainly consists of the accelerated solar wind core electrons, we would expect the hot population to exhibit higher temperatures than the undisturbed solar wind core electrons. Based on our analysis, the solar wind core temperature is systematically around $\approx 15 \mathrm{eV}$ lower than the hot population temperature as expected.

The acceleration by ambipolar electric field is not able to explain the hot population energies that extends above $100 \mathrm{eV}$. However, the high-energy tail of the distribution only has a minor contribution to the total density of the population and the tail can be explained to be the suprathermal component of the solar wind electron distribution.

Madanian et al. (2016b) studied the DEF for energies inside and outside diamagnetic cavity in July 2015 and found that there is a drop in the electron energy spectra for energies $40 \mathrm{eV}$ to some hundreds of eV. The authors suggested that the lowered fluxes inside the cavity could be caused by lacking of solar wind electrons that would be prevented from crossing the diamagnetic cavity boundary by some unknown mechanism. To support their claim, the authors simulated the electron differential flux to show that electrons caused by photoionization are not able to explain the observed flux between 40 and $90 \mathrm{eV}$ without including solar wind electrons. The findings of Madanian et al. (2016b) supported the claim that the hot population electrons would originate from external source.

However, while simulations by Deca et al. (2017) considered low outgassing rate (i.e., conditions at large heliocentric distances), Madanian et al. (2016a) studied electron fluxes near perihelion when the induced ionosphere was already formed. Hence, the mechanism that accelerates the external electrons might be different at large heliocentric distances and near perihelion. Further studies of the acceleration processes near perihelion are needed to identify the dominant mechanism.

Warm population density is always much higher than local solar wind density and it behaves fairly similarly to the total electron density as a function of cometocentric distance. Warm population density forms a fraction of the total electron density. Hence, the warm population seen by RPC-IES seems to consist of mostly electrons that are born during the ionization process (i.e., bulk electrons), although only part of this population is directly detected by RPC-IES.

\section{Conclusions}

We studied the electron populations measured by RPC-IES near comet 67P from 2014 to September 2016. A double-kappa function adopted from Broiles et al. (2016a) has been fitted to electron distribution to study the evolution of the populations, known as warm and hot, as a function of heliocentric distance. We extracted estimates for the electron densities and temperatures for both populations and validated the results using measurements from other RPC instruments.

We updated the warm and hot population density values given by Broiles et al. (2016a). The fitted warm population density varies between 100 and $30 \mathrm{~cm}^{-3}$ over the heliocentric distance range and the hot population varies from 3 to $0.08 \mathrm{~cm}^{-3}$. While the hot population density is comparable with thermal solar wind core electron densities, the warm population is several times denser than the core electrons.

We observed that when the cometary neutral outgassing rate is high (i.e., near perihelion) the suprathermal electrons are 
well characterized by double-kappa distribution, while at large heliocentric distances the kappa functions fit during the worst months only around $10 \%$ of the time.

The hot population density increased from $\approx 0.1$ to $\approx 3 \mathrm{~cm}^{-3}$ between 3.5 and $1.3 \mathrm{AU}$ while the temperature increased only $33 \%$. Warm population properties varied less as a function of heliocentric distance compared to the hot population. However, the warm population density is shown to be inversely dependent on the radial distance from the comet surface similarly to the total electron density (Edberg et al. 2015; Galand et al. 2016). If the cometocentric distance dependence for the warm population is taken into account, the difference between the warm population density near perihelion and beyond $3 \mathrm{AU}$ is nearly as large as in the case of hot population density.

The temperatures of either hot or warm electron populations seen by RPC-IES do not show any significant dependence on the radial distance from the cometary surface.

The warm population seen by RPC-IES consists of partly cometary electrons that are produced during the ionization process and that have not undergone any major acceleration as well as accelerated electrons that form the high-energy part of the distribution. The acceleration/heating mechanism for the warm electrons remains unknown.

On the other hand, we show evidence against the hypothesis that the hot population consists solely of solar wind halo electrons. However, the hot population is suggested to originate from an external source such as solar wind and mainly to consist of an accelerated core electron population. At large heliocentric distances the solar wind core electrons can be accelerated by the ambipolar electric field in the close comet plasma environment but further knowledge of the acceleration process near perihelion is needed. The high-energy tail $(>100 \mathrm{eV})$ of the hot population is expected to contain the solar wind suprathermal electrons (i.e., halo and strahl electrons).

Our study mainly focuses on times when the comet is not interacting with large-scale solar wind structures such as CIRs, thus more work is needed to understand how the electron populations are influenced by such solar events.

Acknowledgements. Rosetta is an ESA mission with contributions from the member states and from NASA. Work at LPC2E is supported by ESEP, CNES, and by ANR under the financial agreement ANR-15-CE31-0009-01. Part of this work was inspired by discussions within International Team 336: "Plasma Surface Interactions with Airless Bodies in Space and the Laboratory" and Team 402: "On the plasma environment of comet 67P after Rosetta" at the International Space Science Institute, Bern, Switzerland. Datasets of the RPC-IES instrument have been downloaded from the ESA Planetary Science Archive (http://archives.esac.esa.int/psa). The authors acknowledge the
Principal Investigator J. Burch of the RPC-IES instrument onboard the Rosetta mission for providing datasets in the archive.

\section{References}

Beth, A., Galand, M., \& Heritier, K. 2019, A\&A, 630, A47 (Rosetta 2 SI) Broiles, T. W., Livadiotis, G., Burch, J. L., et al. 2016a, J. Geophys. Res. Space Phys., 1217407

Broiles, T. W., Burch, J. L., Chae, K., et al. 2016b, MNRAS, 462, S312

Burch, J. L., Goldstein, R., Cravens, T. E., et al. 2007, Space Sci. Rev., 128, 697

Carr, C., Cupido, E., Lee, C. G. Y., et al. 2007, Space Sci. Rev., 128, 629

Clark, G., Broiles, T. W., Burch, J. L., et al. 2015, A\&A, 583, A24

Deca, J., Divin, A., Henri, P., et al. 2017, Phys. Rev. Lett., 118, 20510

Edberg, N. J., Eriksson, A. I., Odelstad, E., et al. 2015, Geophys. Res. Lett., 42 4263

Edberg, N. J., Eriksson, A. I., Odelstad, E., et al. 2016a, J. Geophys. Res. Space Phys., 121, 949

Edberg, N. J., Alho, M., André, M., et al. 2016b, MNRAS, 462, S45

Engelhardt, I. A., Eriksson, A. I., Vigren, E., et al. 2018, A\&A, 616, A51

Eriksson, A. I., Boström, R., Gill, R. B., et al. 2007, Space Sci. Rev., 128, 729

Eriksson, A. I., Engelhardt, I. A., André, M., et al. 2017, A\&A, 605, A15

Feldman, W. C., Asbridge, J. R., Bame, S. J., Montgomery, M. D. \& Gary, S. P. 1975, J. Geophys. Res., 80, 4181

Fitzenreiter, R. J., Ogilvie, K. W., Chornay, D. J., \& Keller, J. 1998, Geophys. Res. Lett., 25, 249

Galand, M., Héritier, K. L., Odelstad, E., et al. 2016, MNRAS, 462, S331

Gasc, S., Altwegg, K., Balsiger, H., et al. 2017, MNRAS, 469, S108

Gilet, N., Henri, P., Wattieaux, G., Cilibrasi, M., \& Béghin, C. 2017, Radio Sci., 52,1432

Hässig, M., Altwegg, K., Balsiger, H., et al. 2015, Science, 347, aaa0276

Hajra, R., Henri, P., Myllys, M. C., et al. 2018, MNRAS, 480, 4544

Hansen, K. C., Altwegg, K., Berthelier, J. J., et al. 2016, MNRAS, 462, S491

Heritier, K. L., 2018, Space and Atmospheric Physics Group (SPAT), PhD Thesis, Imperial College London, UK

Heritier, K. L., Henri, P., Vallières, X., et al. 2017, MNRAS, 469, S118

Heritier, K. L., Galand, M., Henri, P., et al. 2018, A\&A, 618, A77

Johansson, F. L., Odelstad, E., Paulsson, J. J. P. F., et al. 2017, MNRAS, 469, S626

Livadiotis, G., \& McComas, D. J. 2011, ApJ, 741, 88

Madanian, H., Cravens, T. E., Rahmati, A., et al. 2016a J. Geophys. Res. Space Phys., 121, 5815

Madanian, H., Cravens, T. E., Burch, J., et al. 2016b, AJ, 153, 30

Mandt, K. E., Eriksson, A., Edberg, N. J., et al. 2016, MNRAS, 462, S9

Odelstad, E., Eriksson, A. I., Edberg, N. J., et al. 2015, Geophys. Res. Lett., 42, 10

Odelstad, E., Stenberg-Wieser, G., Wieser, M., et al. 2017, MNRAS, 469, S568

Odelstad, E., Eriksson, A. I., Johansson, F. L., et al. 2018, J. Geophys. Res. Space Phys., 123, 7, 5870

Pierrard, V., Lazar, M., Poedts, S., et al. 2016, Sol. Phys., 291, 2165

Trotignon, J. G., Michau, J. L., Lagoutte, D., et al. 2007, Space Sci. Rev., 128, 713

Štverák, Š., Maksimovic, M., Trávníček, P. M., et al. 2009, J. Geophys. Res. Space Phys., 114, A5

Simon Wedlund, C., Kallio, E., Alho, J., et al. 2016, A\&A, 587, A154 


\section{Appendix A: RPC-LAP temperature estimate}

The RPC-LAP instrument investigated the bulk properties of the cometary plasma, including the effective electron temperature. We only provide a brief description as background to interpretation of the RPC-LAP data and refer to Eriksson et al. (2017) for more details.

As only electrons of sufficient energy can reach a negatively charged surface, the electron current to a spherical probe at potential $V_{\mathrm{p}}$ with respect to infinity is

$I_{\mathrm{e}}=I_{\mathrm{e} 0} \exp \left(V_{\mathrm{p}} / k_{\mathrm{B}} T_{\mathrm{e}}\right)$,

where $I_{\mathrm{e} 0}$ is the random thermal current to a sphere in a plasma (proportional to electron density), $T_{\mathrm{e}}$ is the electron temperature of the plasma, and other constants have their usual meaning. If a single Maxwellian is not a sufficient description of the electron gas, several terms of the same form but different $I_{\mathrm{e} 0}$ and $T_{\mathrm{e}}$ can be added.

At intervals of typically a few minutes, the RPC-LAP probes were swept in potential $V_{\mathrm{p}}$ and the probe current was measured and transmitted to ground, where they are analyzed by an automated algorithm. For each sweep, the spacecraft potential is determined and thereby the voltage range over which Eq. (A.1) also holds. Current contributions from ions (Odelstad et al. 2018) and photoelectron emission (Johansson et al. 2017) are also identified and subtracted from the total current by an automated algorithm. The remaining current should then represent $I_{\mathrm{e}}$, and the algorithm then derives $T_{\mathrm{e}}$ by a linear least-squares fit of the logarithm of the remaining current to the voltage.

Several complications can arise in this procedure, including uncertainty in the determination of spacecraft potential, photoelectron current, and ion current, temporal variations of the plasma during the sweep (typically a few seconds), and the presence of several electron populations. An example of the latter case is the cold electron gas $(T \approx 0.1 \mathrm{eV})$ often found at the same time as a warm population $(T=5-15 \mathrm{eV})$ in the RPC-LAP data (Eriksson et al. 2017). As seen in Eq. (A.1), the current contribution from the cold population falls off rapidly with increasingly negative potential, so our fitted $T_{\mathrm{e}}$ value mainly refers to the warm electrons. The cold electrons can be studied by a different method combining RPC-LAP and RPC-MIP data (Engelhardt et al. 2018) and by a completely independent approach using RPC-MIP alone (Gilet et al. 2017).

\section{Appendix B: Validation of the fitting routine}

To validate our fitting routine, we compared the achieved RPCIES electron moments with the measurements carried out by other RPC instruments. We used the temperature estimates from the RPC-LAP (Eriksson et al. 2007) and the density estimates from the RPC-MIP (Trotignon et al. 2007). In addition, we used an alternative method to extract the density estimates from the RPC-IES electron data. The method is to integrate the plasma moments over energy.

The RPC-LAP instrument examined the effective electron temperature that is suitable for comparison with the warm population temperature defined from the RPC-IES data. While the observations by the RPC-LAP are affected by all the bulk electrons, the RPC-IES has a low-energy threshold $(>4.32 \mathrm{eV})$ that depends on the spacecraft potential. Thus, most of the time the RPC-IES observes only part of the bulk electrons. Thus, the RPC-IES warm population temperature can be higher
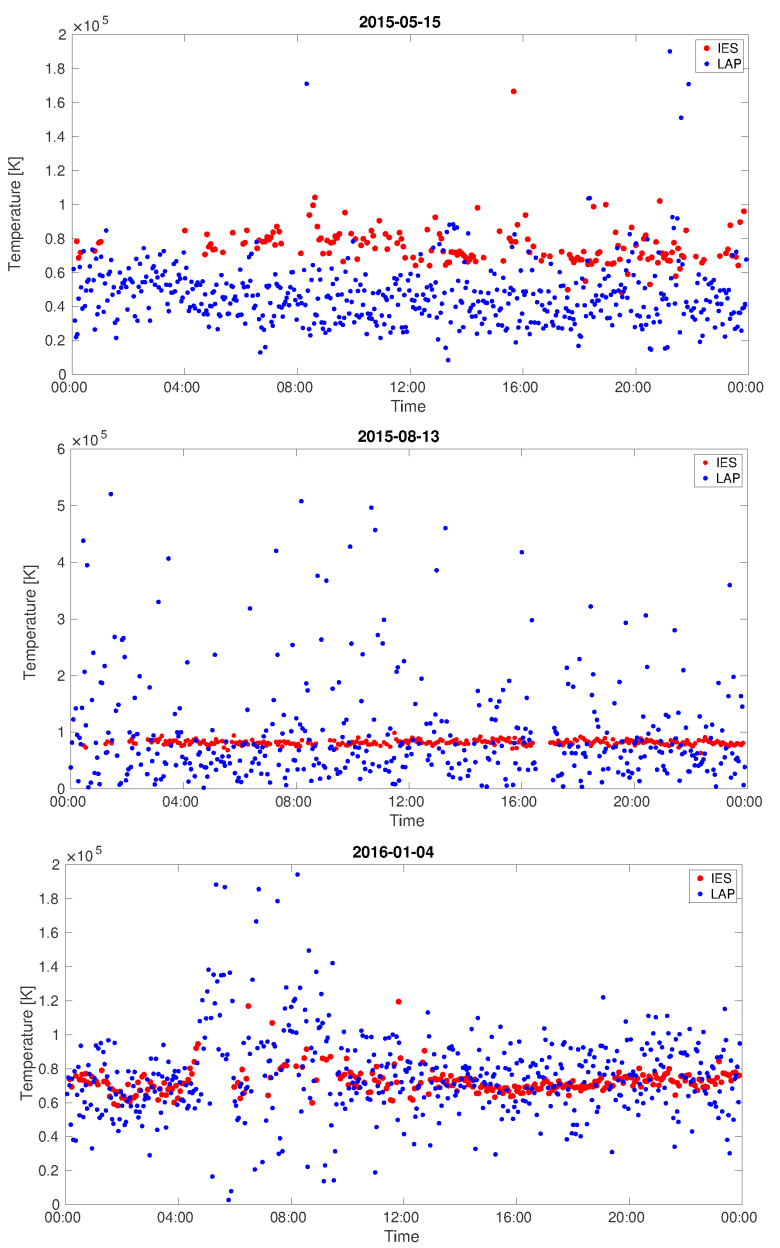

Fig. B.1. RPC-IES warm population and RPC-LAP temperature as a function of time. Each row shows a different day. Uppermost panel: time interval before perihelion; middle: during perihelion; and lower panel: after perihelion.

than the bulk electron temperature seen by the RPC-LAP. The other reason why the warm population is expected to have higher temperature at times than the bulk electrons is the existence of the cold electron population. The cold electrons has been cooled down owing to collisions with neutrals near the comet nucleus (Eriksson et al. 2017; Engelhardt et al. 2018).

Figure B.1 shows the temperatures given by the RPC-LAP (blue points) and the fitted warm population temperatures (red points) for three one-day intervals (2015-05-15, 2015-08-13, and 2016-01-04). The spacecraft potential during the corresponding time intervals are shown in Fig. B.2 to give an idea of its variations. The uppermost panels show the time interval before perihelion passage, the middle panels during perihelion, and the lower panels after perihelion. The RPC-LAP has higher time resolution than the RPC-IES and its temperature values can vary during the RPC-IES measurement interval.

In addition to Fig. B.1, that is comparing the RPC-LAP and the RPC-IES temperatures during one-day intervals, we computed daily median values of the warm population temperature. The same treatment was done to the RPC-LAP temperature. The results are shown in Fig. B.3 as a function of time. The blue points show the RPC-LAP measurements and the red points indicate warm population temperatures seen by RPC-IES. Figure B.3 clearly shows that the RPC-IES and RPC-LAP temperatures are the same order of magnitude even though intermittently the 


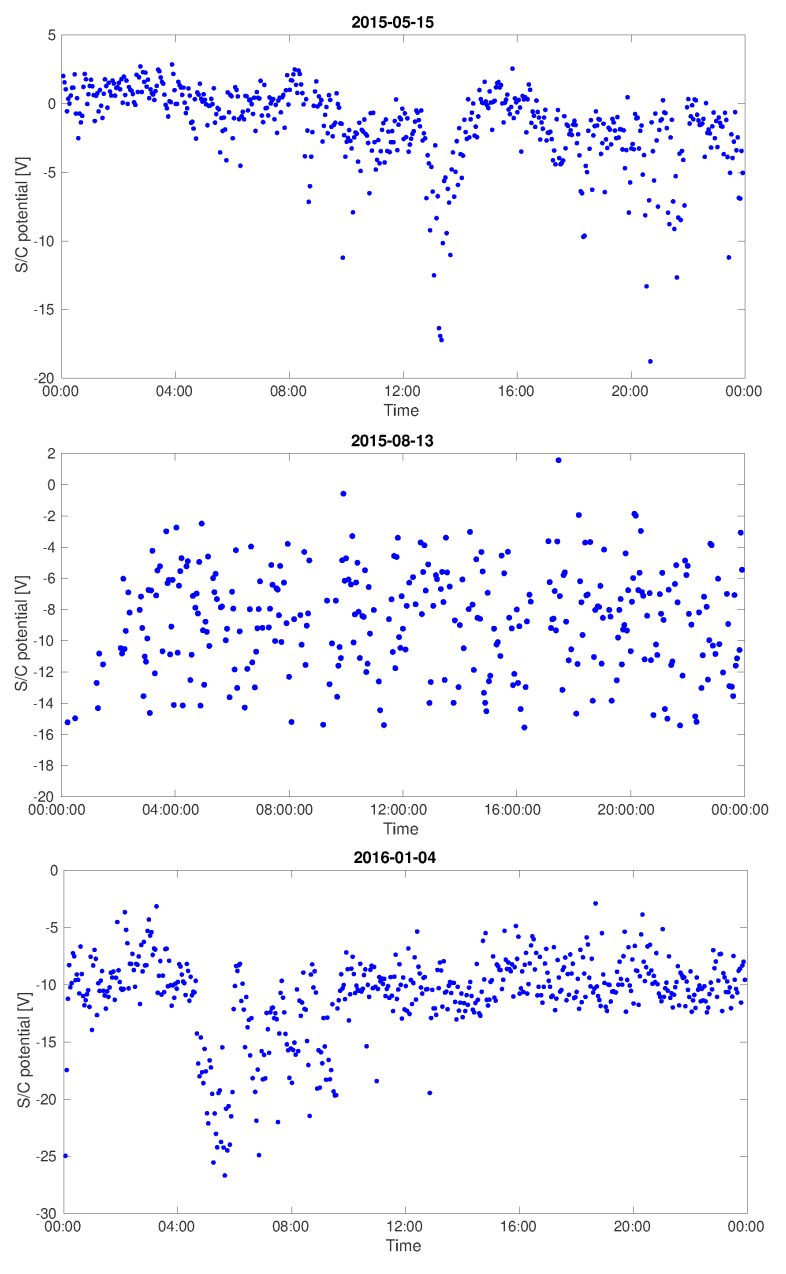

Fig. B.2. Spacecraft potential as a function of time.

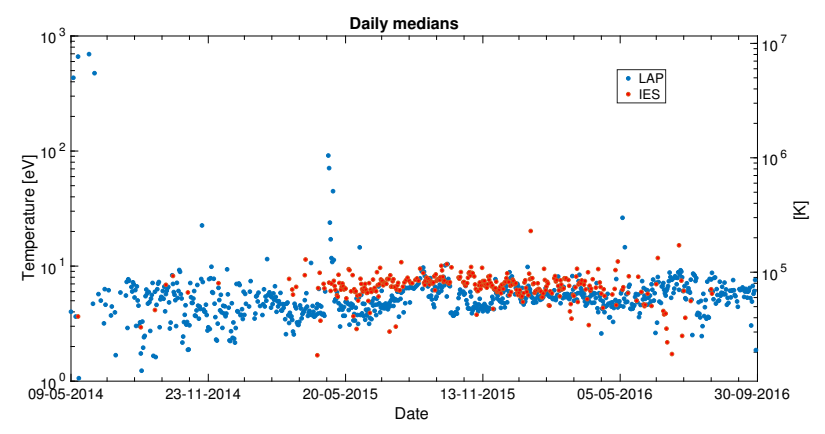

Fig. B.3. Daily median temperature estimated by RPC-LAP and the fitted warm population temperature by RPC-IES as a function of time.

RPC-IES warm temperature is slightly higher than the RPC-LAP temperature.

Sometimes the fitting routine failed to give good estimates for the plasma parameters. We chose not to compute a daily median temperature when the number of values is not considered high enough to be significant. This is why 2014 as well as the end of 2016 are lacking of daily median temperatures from RPC-IES (Fig. B.3). The reasons why there are fewer warm population temperature estimates in 2014 and 2016 compared to 2015 are discussed in Sect. 3.3 in the main article.

The RPC-MIP measured the total plasma density and thus, these measurements are ideal for warm population density validation. The RPC-MIP instrument gave the density for the
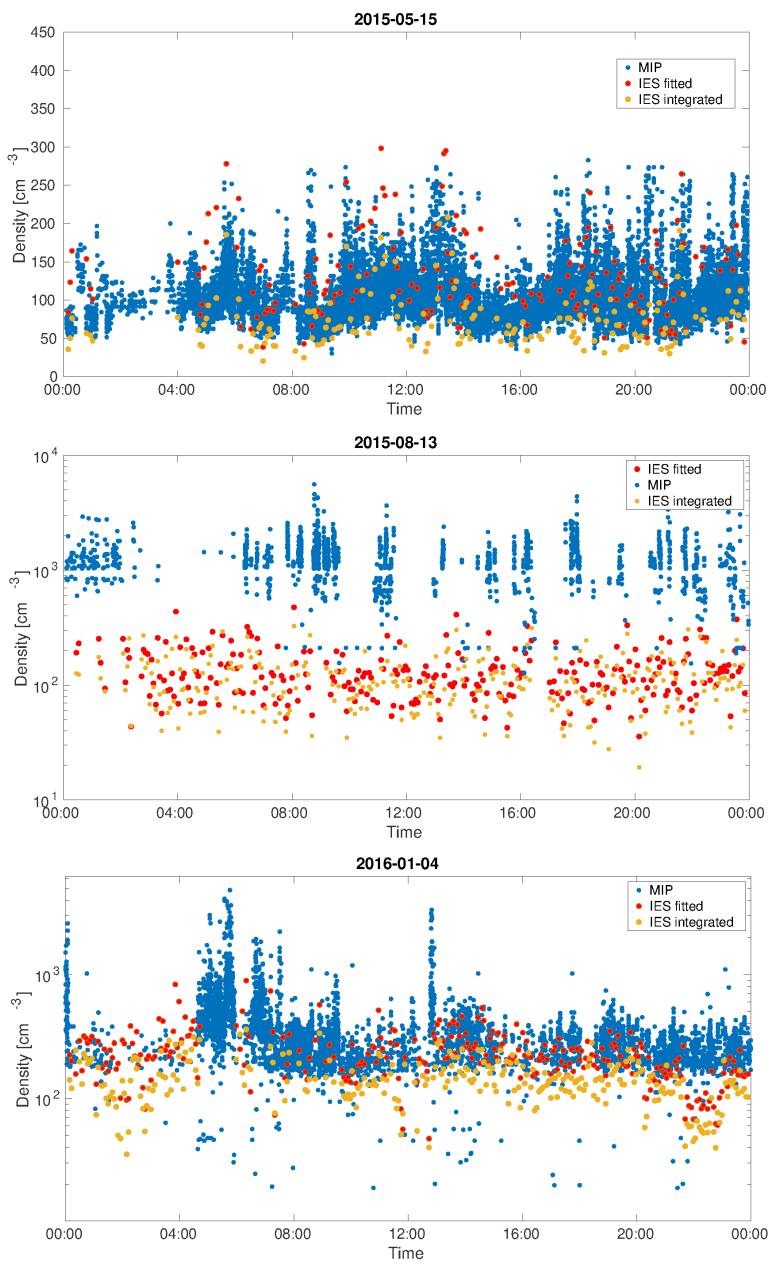

Fig. B.4. RPC-IES and RPC-MIP densities as a function of time. The red points show the fitted density values for the warm population while the yellow points represent the integrated (starting at energy $10.8 \mathrm{eV}$ ) RPC-IES densities. The time intervals are the same as in the Fig. B.1.

cold and bulk electrons combined, while the RPC-IES gave the density only for electrons exceeding the lowest energy threshold of the instrument. Thus, the warm population density derived from the RPC-IES should be less or equal to that of RPC-MIP. The comparison between the RPC-MIP (blue) and warm population (red) densities are shown in Fig. B.4. The time intervals are the same as in Fig. B.1.

During 2015-05-15 and 2016-01-04, when the distance from the Sun was around 1.65 AU and 2.05 AU, the RPC-IES and the RPC-MIP densities match, while near perihelion (at 1.25 AU) the difference between the densities is notable. Close to perihelion on August 2015, RPC-LAP observed that very high fraction of sweeps contained cold electrons (Engelhardt et al. 2018), which can explain the difference in the densities.

We used the cometary flybys during February 2015 in Sect. 4.2 in the main article to study the cometocentric distance dependence of the warm population density (Fig. 5). In Fig. B.5 we show how the RPC-IES warm population density corresponds to the RPC-MIP density during the flybys. Both instruments gave densities that are of the same order of magnitude except that the RPC-MIP gave lower values when the altitude is below $50 \mathrm{~km}$. This is because the instrumental mode was used by the RPC-MIP during the observed time interval. 


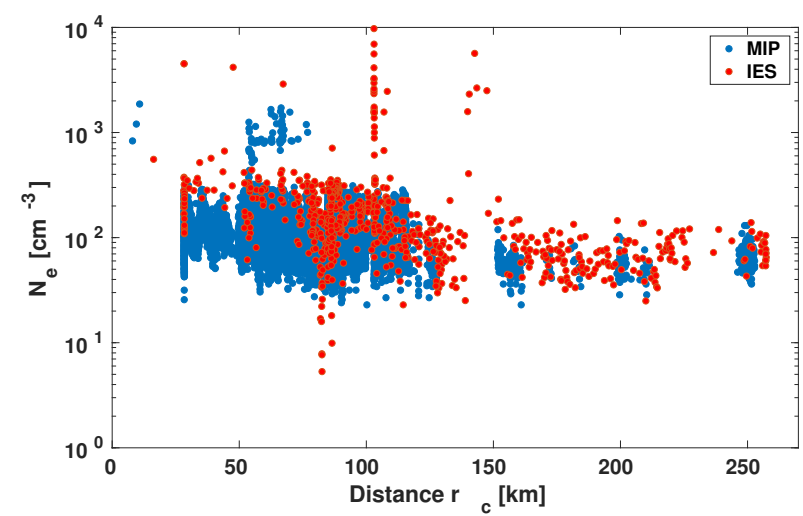

Fig. B.5. RPC-IES warm population and RPC-MIP densities as a function of cometocentric distance during February 2015.

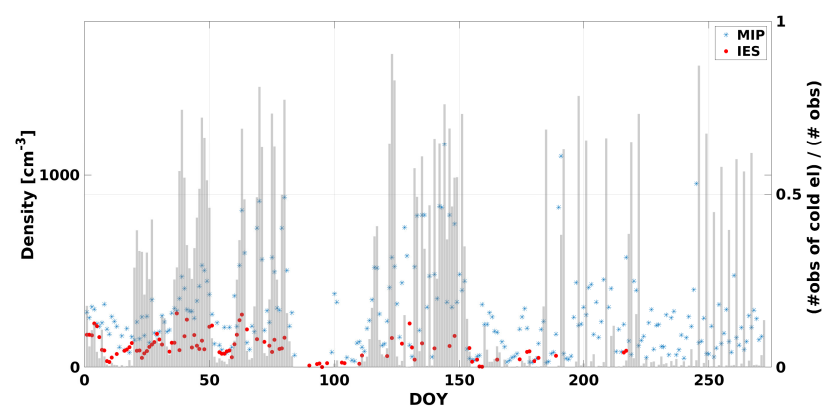

Fig. B.6. Daily median electron densities as a function of the day of the year for 2016. The blue points show measurements from RPC-MIP instrument and the red points indicate those from RPC-IES. The histograms on the background show the ratio of cold electron observations to all observations.

We also compare the daily median densities. Figure B.6 shows the daily median densities from the RPC-MIP and daily medians of warm population density as a function of time for the year 2016. The red points correspond to the RPC-IES measurements and the blue points to the RPC-MIP. The RPC-IES warm population density fulfills the criterion of being lower or equal to the RPC-MIP density.

The histogram in Fig. B.6 shows the ratio of cold electron observations to total number of measured RPC-MIP spectra. The high ratio refers to increased cold population density. Times when there is large difference between the RPC-IES warm population density and RPC-MIP electron density are typically those that have a high fraction of cold electron measurements as expected. The identification of cold electrons from the RPC-MIP mutual impedance spectra are explained by Gilet et al. (2017).

Figure B.4 also shows electron densities that have been integrated (yellow points) using the RPC-IES observations. The integration of the density always starts at energy $10.8 \mathrm{eV}$ while the fitting routine uses the whole available RPC-IES energy range. But because for the majority of the time the spacecraft potential was between -5 and $-10 \mathrm{~V}$ (Odelstad et al. 2015) the RPC-IES was very rarely able to observe electrons below $10 \mathrm{eV}$. Thus, the error of the integration would increase if the integration started from $4.32 \mathrm{eV}$.

The RPC-IES electron particle flux has been corrected for spacecraft potential before the integration. If the absolute value of the spacecraft potential is larger than $10.8 \mathrm{~V}$, it is not possible to retrieve the electron flux between the lower limit of the integration and the spacecraft potential. Thus, the flux is extrapolated assuming a constant for the missing part using a value that equals that at the lowest energy bin when the spacecraft potential is taken into account (see details in Sect. 3.4.3 by Galand et al. 2016). If there is no estimate for the spacecraft potential available or it is below $-16 \mathrm{~V}$, the integration has not been done.

While the RPC-IES measurements are corrected with the spacecraft potential before the integration, the fitting routine does not correct the measurements themselves but includes the spacecraft potential correction to the fitted model distribution (see Eq. (3)). Appendix C gives a more detailed description of the spacecraft potential correction. As can be seen in Fig. B.4, the fitting and integration give comparable values (Fig. B.4). The integrated values are slightly lower than the warm population density because the integration has been started at $10.8 \mathrm{eV}$ while the warm population has usually a peak below $10 \mathrm{eV}$.

\section{Appendix C: Comparison of phase-space density measured by RPC-IES with and without spacecraft potential correction}

The RPC-IES measurements are affected by the spacecraft potential. The negative potential repels electrons reaching the instrument. Thus, the spacecraft potential can increase the lowest energy threshold of the sensor (Galand et al. 2016). If the RPC-IES is measuring energy $E$ for the electrons, the real energy of the electrons in "free space" is $E-V_{\mathrm{SC}}$, where $V_{\mathrm{SC}}$ is the spacecraft potential.

Thus, to get the electron phase-space density in free space the RPC-IES measurements need to be shifted in energy based on the spacecraft potential value and the free-space values for the electron fluxes need to be computed. A detailed description of the spacecraft potential corrections for the RPC-IES measurements is given by Galand et al. (2016).

Figure C.1 demonstrates how the measured phase-space density looks before (blue points) and after (red points) the spacecraft potential correction.

In the present study, we carried out the fitting using the RPCIES measurements without the spacecraft potential correction to the electron flux and instead we included the potential correction

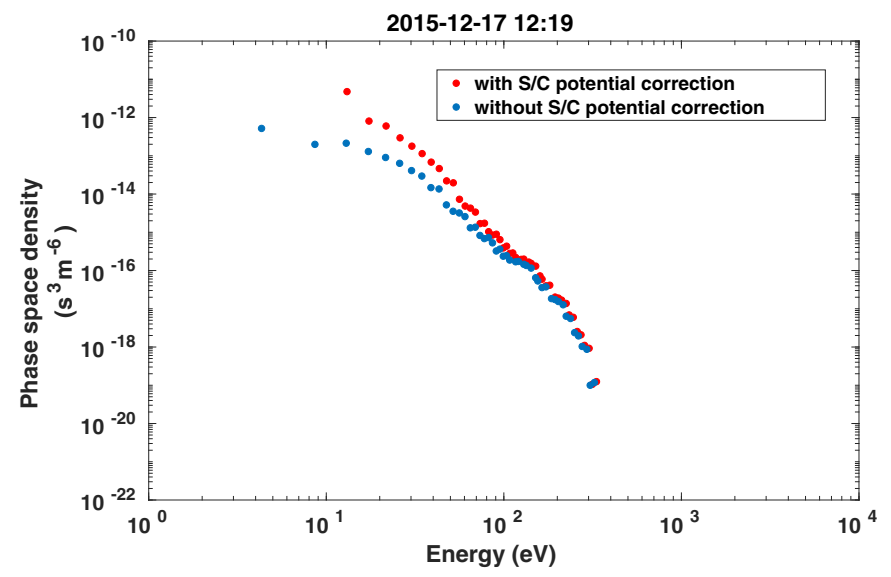

Fig. C.1. Phase-space density measured by RPC-IES with (red) and without (blue) spacecraft potential correction. The red points are shifted in energy and the electron fluxes have been corrected using the method by Galand et al. (2016). 
to the model double-kappa function that has been fitted to the measurements (See Eq. (6)).

In Fig. C.2 we show the measured phase-space density without spacecraft correction to the electron flux (black dots) and the fitted double-kappa function (black line) for one RPC-IES spectrum. The red points show the spacecraft potential corrected phase-space density determined using the method by Galand et al. (2016). The fitted double-kappa function has also been plotted by setting the spacecraft potential term to zero (red line) to demonstrate that it fits to the corrected phase -space density measurements as well. Both the black and red points have been shifted in energy taking into account the spacecraft potential.

Hence, doing the fitting using RPC-IES phase-space density that is corrected for non-spacecraft potential, and including the spacecraft potential correction to the fitted distribution, is equivalent to the approach in which the RPC-IES measurements are spacecraft potential corrected at first and double kappa is fitted by setting the spacecraft potential term to zero. In our study we selected the first approach because it is easier from point of view of the fitting.

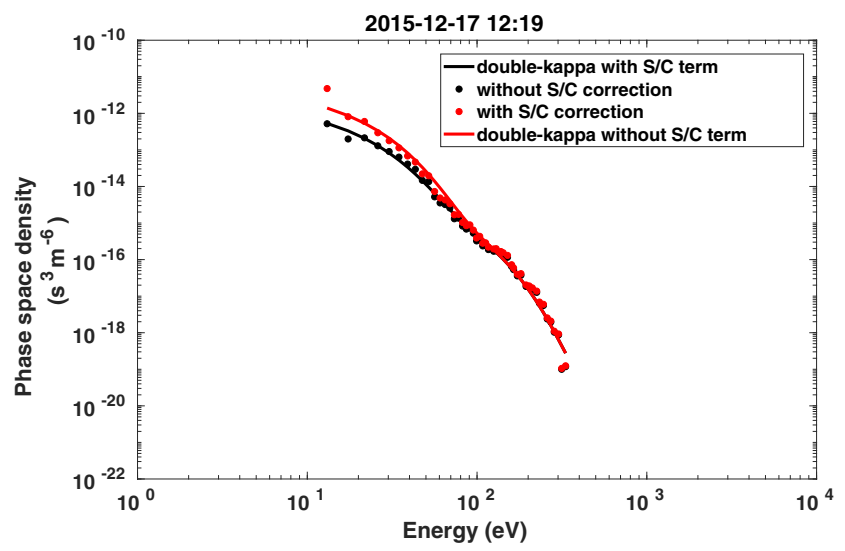

Fig. C.2. Phase-space density with (red dots) and without (black dots) spacecraft potential corrections. Both points have been shifted in energy based on the spacecraft potential value. The black line indicates the double-kappa function including the spacecraft potential term and the red curve indicates the double-kappa without the spacecraft potential term. 